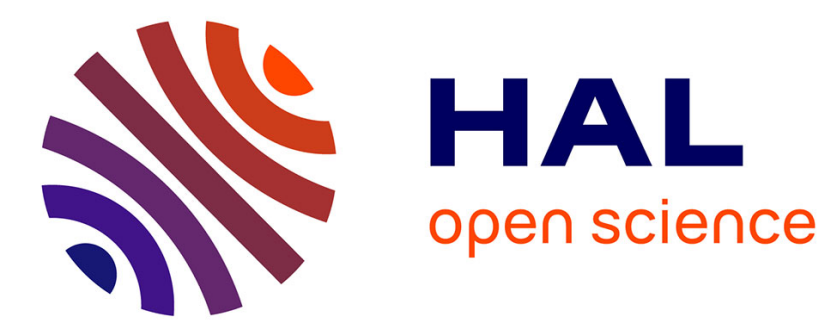

\title{
Magnetic noise of a frozen ferrofluid
}

K. Komatsu, D. L'Hote, S. Nakamae, F. Ladieu, V. Mosser, A. Kerlain, M. Konczykowski, Emeric Dubois, V. Dupuis, R. Perzynski

\section{To cite this version:}

K. Komatsu, D. L'Hote, S. Nakamae, F. Ladieu, V. Mosser, et al.. Magnetic noise of a frozen ferrofluid. Journal of Applied Physics, 2010, 107, pp.09E140. 10.1063/1.3366613 . cea-01394919

\section{HAL Id: cea-01394919 https://hal-cea.archives-ouvertes.fr/cea-01394919}

Submitted on 10 Nov 2016

HAL is a multi-disciplinary open access archive for the deposit and dissemination of scientific research documents, whether they are published or not. The documents may come from teaching and research institutions in France or abroad, or from public or private research centers.
L'archive ouverte pluridisciplinaire HAL, est destinée au dépôt et à la diffusion de documents scientifiques de niveau recherche, publiés ou non, émanant des établissements d'enseignement et de recherche français ou étrangers, des laboratoires publics ou privés. 


\title{
Magnetic noise of a frozen ferrofluid
}

\author{
K. Komatsu, ${ }^{1, a)}$ D. L'Hôte, ${ }^{1}$ S. Nakamae, ${ }^{1}$ F. Ladieu, ${ }^{1}$ V. Mosser, ${ }^{2}$ A. Kerlain, ${ }^{2}$ \\ M. Konczykowski, ${ }^{3}$ E. Dubois, ${ }^{4}$ V. Dupuis, ${ }^{4}$ and R. Perzynski ${ }^{4}$ \\ ${ }^{1}$ Service de Physique de l'Etat Condensé (CNRS/MIPPU/URA 2464), DSM/IRAMIS/SPEC, CEA Saclay, F- \\ 91191 Gif/Yvette Cedex, France \\ ${ }^{2}$ ITRON SAS, 76 avenue Pierre Brossolette, F-92240 Malakoff, France \\ ${ }^{3}$ Laboratoire des Solides Irradiés, Ecole Polytechnique, CNRS-UMR 7642 \& CEA/DSM/IRAMIS, 91128 \\ Palaiseau, France \\ ${ }^{4}$ Laboratoire PECSA, UMR 7195 CNRS, Université Pierre et Marie Curie, -4 place Jussieu, Boîte 51, \\ 75252 Paris Cedex 05, France
}

(Presented 21 January 2010; received 30 October 2009; accepted 15 December 2009; published online 6 May 2010)

\begin{abstract}
The magnetic noise of a frozen ferrofluid made of maghemite nanoparticles dispersed in glycerin is measured using a two-dimensional electron gas based quantum well Hall sensor (QWHS) with the spinning current technique. The frozen ferrofluid shows a superspin glass transition at $67 \mathrm{~K}$. Below this glass transition temperature, the relation between the imaginary part of the ac susceptibility of a bulk ferrofluid sample and the magnetic noise measured with the QWHS gives an indication of a violation of the fluctuation dissipation theorem. () 2010 American Institute of Physics. [doi:10.1063/1.3366613]
\end{abstract}

\section{INTRODUCTION}

Understanding the collective behavior of nanomagnet assemblies is an important problem for statistical physics as well as for applications such as magnetic storage, spintronics, magnetic sensors, and biomedicine. Magnetic noise measurement is a remarkably useful way to understand the microscopic dynamics of interacting nanomagnets because it directly reflects the fluctuations of the magnetic moments. At equilibrium, the magnetic fluctuations are related to the response to a small field through the fluctuation-dissipation theorem (FDT): $\chi^{\prime \prime} \propto S f / T$, where $\chi^{\prime \prime}$ is the dissipative part of the magnetic susceptibility, $S$ is the magnetic noise power spectrum, $f$ is the measurement frequency, and $T$ is the temperature. A frozen "ferrofluid" consists of monodomain nanomagnets suspended in a solid matrix (in our case, frozen glycerin). The individual nanomagnets have a large magnetic moment of the order of $\sim 10^{4} \mu_{\mathrm{B}}$, and thus it is called "superspin." When the nanomagnets are sufficiently concentrated at low enough temperature, long-range dipolar interactions among them produce spin-glass behavior (aging, memory, etc.). 'The system is thus called a "superspin glass."

In the $1990 \mathrm{~s}$, theoretical investigations of the spin and structural glasses relaxation through dynamical studies started and allowed to make some predictions on the nature of the violations of FDT, which could be expected in such out-of-equilibrium systems. ${ }^{2}$ In particular, the concept of "effective temperature" $\left(T_{\text {eff }}\right)$, which allows to extend the FDT to the off-equilibrium state was proposed. It is defined by $\chi^{\prime \prime} \propto S f / T_{\text {eff }}$, with the same proportionality factor as that at equilibrium.

These FDT violations have also been experimentally investigated for a few structural glasses ${ }^{3}$ and one spin glass

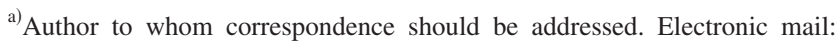
katsuyoshi.komatsu@cea.fr. system $^{4}$ at temperatures lower than the glass transition temperature where the system is off equilibrium. The system then shows aging, which is an evolution toward its equilibrium state with time. Although spin glass is one of the most studied glassy systems, the FDT violation in spin glasses has been measured for only one system due to the difficulty of detecting extremely small noises $\sim 10^{-7} \mathrm{G}^{4}$ To overcome this problem, we have measured the magnetic noise of a frozen ferrofluid in the superspin glass state with a high resolution two-dimensional electron gas (2DEG) based quantum well Hall sensor (QWHS). The left panel in Fig. 1 shows the optical micrograph of the QWHS. Note two advantages of using a superspin glass and QWHS. First the large susceptibility (real part of ac susceptibility $\chi^{\prime}$ and imaginary part of ac susceptibility $\chi^{\prime \prime}$ ) of superspin glasses ${ }^{1}$ ensures a large noise power. Second the distance from the 2DEG layer to the sample is $\sim 1 \mu \mathrm{m}$, thus the measured magnetic noise is much larger than that of the bulk sample measurements using superconducting quantum interference device (SQUID) sensor because the magnetic field of the nanomagnets decreases with the distance $r$ as $1 / r^{3} .5$

\section{EXPERIMENT}

We use a QWHS based on pseudomorphic AlGaAs/ InGaAs/GaAs heterostructures. ${ }^{6-8}$ The nominal Hall cross

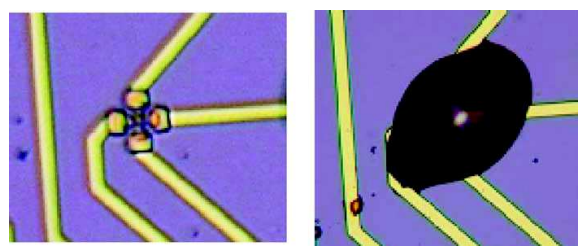

FIG. 1. (Color online) Left panel: Optical micrograph of the 2DEG Hall microsensor. Right panel: Optical micrograph of the same microsensor with the ferrofluid drop on its surface. 

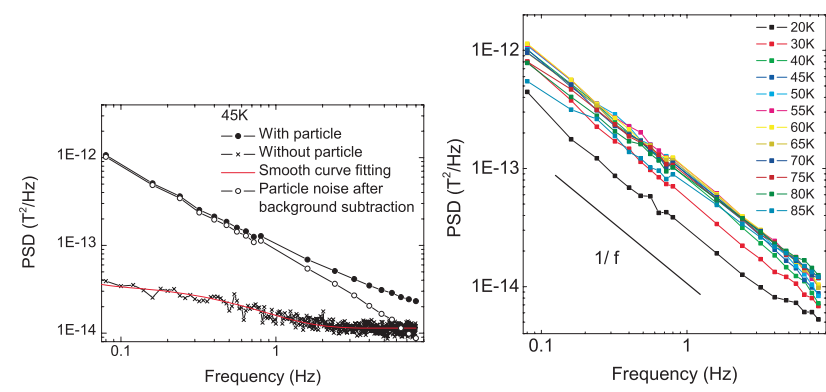

FIG. 2. (Color online) Left panel: Power spectrum density of the frozen ferrofluid (open symbols) obtained by subtracting the Hall probe noise (cross symbol) from the power spectrum density which contains both of them (filled symbol), as a function of frequency $f$, at $45 \mathrm{~K}$ in zero applied field. Right panel: The power spectrum density of the ferrofluid at various temperatures from 20 to $85 \mathrm{~K}$

area of $\sim 2 \times 2 \mu \mathrm{m}^{2}$ (estimated effective area $\sim 1.6$ $\times 1.6 \mu \mathrm{m}^{2}$ ) is located $\sim 1 \mu \mathrm{m}$ (according to the maker's design specification; estimated real distance is $\sim 650 \mathrm{~nm}$ ) beneath the probe surface. We also use the spinning current technique, which simultaneously suppresses the low frequency noise and the Hall voltage offset. This is achieved by switching the current injection and the voltage detection directions at a spinning frequency $f_{\text {spin. }}$ In our experiment, $f_{\text {spin }}=1 \mathrm{kHz}$. The effectiveness of this method has been recently reported by Kerlain and Mosser. ${ }^{8}$ Using this technique, we achieve a sensitivity of $\sim 2 \mathrm{mG} / \sqrt{ } \mathrm{Hz}$ at $0.01 \mathrm{~Hz}$ and at temperatures ranging from 20 to $85 \mathrm{~K}$.

The ferrofluid used in this experiment is made of maghemite $\gamma-\mathrm{Fe}_{2} \mathrm{O}_{3}$ nanoparticles dispersed in glycerin with a volume fraction of $15 \%$, prepared following the method of Ref. 9. The nanoparticles average diameter and magnetic moment are $8.6 \mathrm{~nm}$ and $10^{4} \mu_{\mathrm{B}}$, respectively. A small $200 \mathrm{pl}$ drop of ferrofluid was deposited directly on the probe surface. At low temperatures below $190 \mathrm{~K}$, the fluid (glycerin) is frozen and the only remaining magnetic degree of freedom is that of the particle magnetic moments. These moments (superspins) interact through magnetic dipolar interactions leading to a superspin-glass transition at the temperature, $T_{\mathrm{g}} \approx 67 \mathrm{~K} .{ }^{10}$ The detailed characteristics of the sample can be found in Ref. 10. The dc and ac susceptibilities of the bulk ferrofluid (approximately $1.5 \mu \mathrm{l}$ ) were measured with a commercial SQUID magnetometer.

\section{RESULTS AND DISCUSSION}

Prior to measuring the magnetic fluctuation signal of the frozen ferrofluid, we characterized the Hall voltage noise spectra of a pristine Hall probe at various temperatures. Subsequently, the small drop of the ferrofluid was deposited on the probe and cooled down to temperatures ranging from 20 to $85 \mathrm{~K}$. The right panel of Fig. 1 shows the optical micrograph of the ferrofluid dropped on the surface of the QWHS. The ferrofluid is liquid at room temperature but is frozen by cooling below $190 \mathrm{~K}$.

The left panel of Fig. 2 shows the power spectrum density of the frozen ferrofluid (denoted by open symbols) obtained by subtracting the Hall probe noise (cross symbol) from the total power spectrum density (filled symbol) as a
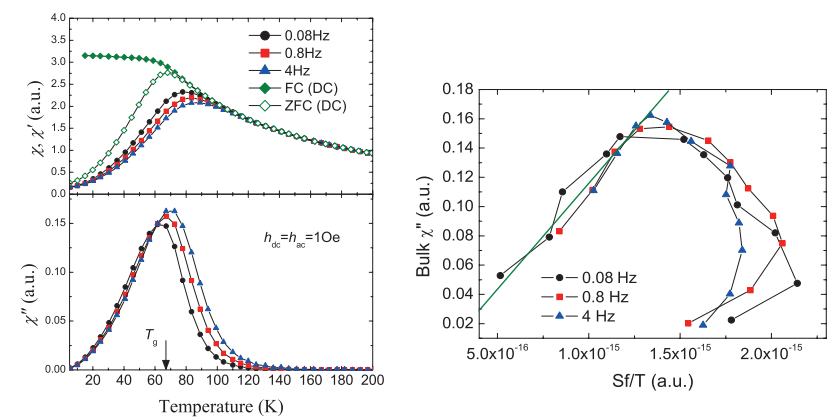

FIG. 3. (Color online) Left panel: Temperature dependence of $\chi$ for dc measurement and $\chi^{\prime}$ and $\chi^{\prime \prime}$ for ac measurement with a SQUID magnetometer. The measurement field was 1 Oe for both $\mathrm{dc}$ and ac measurements. Right panel: $\chi^{\prime \prime}$ of the bulk sample as a function of $S f / T$ measured with the QWHS. The two kinds of measurements have been performed at the same temperature and frequency.

function of frequency $f$, at $45 \mathrm{~K}$ in zero applied field. The right panel of Fig. 2 shows the power spectrum density of the ferrofluid at various temperatures between 20 and $85 \mathrm{~K}$. It exhibits an almost $1 / f$ dependence in the frequency range from 0.08 to $8 \mathrm{~Hz}$. Here the power spectrum density was averaged over about $1 \mathrm{~h}$ after waiting for the temperature stabilization cooling from $85 \mathrm{~K}$ (about $10 \mathrm{~min}$ ). The left panel of Fig. 3 shows the temperature dependence of the magnetic zero-field-cooled and field-cooled susceptibilities $\chi$ for dc measurement and $\chi^{\prime}$ and $\chi^{\prime \prime}$ for ac measurement with a SQUID magnetometer measured at temperatures from 5 to $200 \mathrm{~K}$. The measurement field was $1 \mathrm{Oe}$, which is sufficiently small for the linear response, for both dc and ac measurements. If the power spectrum density $S$, obeys FDT, then $\chi^{\prime \prime} \propto S f / T$ should hold. The right panel of Fig. 3 shows $\chi^{\prime \prime}$ of the bulk sample plotted as a function of $S f / T$ for various temperatures and frequencies. The solid line indicates the linear relation corresponding to $\chi^{\prime \prime} \propto S f / T$, which is true only in the high temperature region above $T_{\mathrm{g}}$, where the system is in the equilibrium (super)paramagnetic state. Interestingly, the plots deviate from this equilibrium line at temperatures below $T_{\mathrm{g}}$ where the system is in the out-of-equilibrium (super)spin glass state. This deviation, therefore, can be considered as an indication of the FDT violation below $T_{\mathrm{g}}$. A beyond-doubt confirmation of the existence of the FDT violation should be obtained through further investigations using various samples, other QWHS's detectors and improved experimental procedures (e.g., waiting time dependence).

\section{SUMMARY}

In summary, using a 2DEG QWHS with spinning current, we succeeded in measuring the magnetic noise of a frozen ferrofluid that shows a superspin glass transition at $T_{\mathrm{g}}=67 \mathrm{~K}$. Below $T_{\mathrm{g}}$, the relation between the imaginary part of the bulk ferrofluid sample ac susceptibility and the magnetic noise measured with the QWHS indicates a possible violation of FDT. Further studies, by this same method, of the nonequilibrium dynamics of the ferrofluid, such as aging effect are under development. 


\section{ACKNOWLEDGMENTS}

This work was supported by the RTRA-Triangle de la Physique (MicroHall).

${ }^{1}$ P. E. Jönsson, Adv. Chem. Phys. 128, 191 (2004); P. E. Jönsson, R. Mathieu, P. Nordblad, H. Yoshino, H. Aruga Katori, and A. Ito, Phys. Rev. B 70, 174402 (2004); D. Parker, V. Dupuis F. Ladieu, J.-P. Bouchaud, E. Dubois, R. Perzynski, and E. Vincent, ibid. 77, 104428 (2008).

${ }^{2}$ L. F. Cugliandolo and J. Kurchan, Phys. Rev. Lett. 71, 173 (1993); C. A. Angell, Science 267, 1924 (1995); L. F. Cugliandolo, J. Kurchan, and L. Peliti, Phys. Rev. E 55, 3898 (1997); A. Barrat, ibid. 57, 3629 (1998); J.-L. Barrat and W. Kob, Europhys. Lett. 46, 637 (1999); D. A. Stariolo and S. A. Cannas, Phys. Rev. B 60, 3013 (1999); F. Sciortino and P. Tartaglia, Phys. Rev. Lett. 86, 107 (2001); H. Kawamura, ibid. 90, 237201 (2003). ${ }^{3}$ T. S. Grigera and N. Israeloff, Phys. Rev. Lett. 83, 5038 (1999); L. Bellon, S. Ciliberto, and C. Laroche, Europhys. Lett. 53, 511 (2001); L. Buisson, S. Ciliberto, and A. Garcimartin, ibid. 63, 603 (2003); B. Abou and F. Gallet, Phys. Rev. Lett. 93, 160603 (2004); N. E. Israeloff, H. Oukris, and P. S. Crider, J. Non-Cryst. Solids 352, 4915 (2006); N. Greinert, T. Wood, and P. Bartlett, Phys. Rev. Lett. 97, 265702 (2006); M. Lucchesi, A. Dominjon, S. Capaccioli, D. Prevosto, and P. A. Rolla, J. Non-Cryst. Solids
352, 4920 (2006); S. Jabbari-Farouji, D. Mizuno, M. Atakhorrami, F. C. Mackintosh, C. F. Schmidt, E. Eiser, G. H. Wegdam, and D. Bonn, Phys. Rev. Lett. 98, 108302 (2007); P. Jop, A. Petrosyan, and S. Ciliberto, Philos. Mag. 88, 4205 (2008)

${ }^{4}$ D. Hérisson and M. Ocio, Phys. Rev. Lett. 88, 257202 (2002).

${ }^{5}$ D. L'Hôte, S. Nakamae, F. Ladieu, V. Mosser, A. Kerlain, and M. Konczykowski, J. Stat. Mech.: Theory and Exp. 2009, P01027.

${ }^{6}$ A. Kerlain and V. Mosser, Sens. Actuators, A 142, 528 (2008).

${ }^{7}$ V. Mosser, G. Jung, J. Przybytek, M. Ocio, and Y. Haddab, Proc. SPIE 5115, 183 (2003); V. Mosser, S. Contreras, S. Aboulhouda, Ph. Lorenzini, F. Kobbi, J. L. Robert, and K. Zekentes, Sens. Actuators, A 43, 135 (1994); N. Haned and M. Missous, Sens Actuators, A 102, 216 (2003); V. P. Kunets, R. Pornraenke, J. Dobbert, H. Kissel, U. Muller, H. Kostial, E. Wiebicke, G. G. Tarasov, Y. I. Mazur, and W. T. Masselink, IEEE Sens. J. 5, 883 (2005).

${ }^{8}$ A. Kerlain and V. Mosser, Sensor Lett. 5, 192 (2007).

${ }^{9}$ R. Massart, IEEE Trans. Magn. 17, 1247 (1981).

${ }^{10}$ E. Wandersman, V. Dupuis, E. Dubois, R. Perzynski, S. Nakamae, and E. Vincent, Europhys. Lett. 84, 37011 (2008); S. Nakamae, Y. Tahri, C. Thibierge, D. L'Hôte, E. Vincent, V. Dupuis, E. Dubois, and R. Perzynski, J. Appl. Phys. 105, 07E318 (2009). 